\title{
Applications of an Ion Beam Cross Section Polisher in Art Conservation and Preservation
}

\author{
G. McMahon*, L. Zajac**, B. Adams-Hebard***, D. Larsen ${ }^{+}$and N. Erdman ${ }^{++}$ \\ * Nanofabrication Cleanroom Facility, Boston College, Newton MA 02459 \\ ** College of Arts and Sciences, Boston College, Chestnut Hill MA 02467 \\ *** Burns Library Conservator, Boston College, Chestnut Hill MA 02467 \\ + McMullen Museum of Art, Boston College, Chestnut Hill MA 02467 \\ ++ JEOL USA, Peabody MA 01960
}

A common issue facing the materials scientist working in the field of art conservation and preservation is the small amount of sample available for study. It follows that there is little room for error in all aspects of a materials research experiment, including sample preparation for microscopic analysis. This is especially true for cross-section analysis at surfaces, where mechanical damage at the surface induced during the polishing process may remove important layers (even in the hands of the most skilled technician), either because they are very thin, or are of poor mechanical integrity. A solution to this problem is the use of an ion beam cross section polisher for the preparation of high quality specimens with no mechanical damage and full preservation of surface layers.

High quality cross section samples have been prepared from a small fragment of a terra cotta bust of the Marquis de Lafayette attributed to Jean-Antoine Houdon (20 March 1741 - 15 July 1828), using the JEOL cross section polisher. The general principle of the instrument is shown schematically in Fig. 1. A broad beam of Ar ions is directed towards the sample with accelerating voltages between 2 and $6 \mathrm{kV}$. A masking plate is used to expose the cross section to be polished while protecting the other surfaces of the sample from the ion beam. During the polishing process, the sample is continuously rotated $+/-30^{\circ}$ to remove crystallographic effects on the polishing rate and ensure an evenly polished surface. The final polished area is typically $500 \mu \mathrm{m}(\mathrm{FWHM})$ in size.

An example of a cross section obtained using this tool comes from the surface of a broken bust of the Marquis de Lafayette attributed to Houdon and held at the McMullen Museum of Art at Boston College (Fig. 2). A low magnification backscattered electron image from the ion beam cross section polished sample is shown in Fig. 3. The bulk of material at the bottom of the image is the terra cotta, but the layers of the surface finish are of the most interest. Further microscopic and EDS analysis was performed in these regions to search for further clues as to the provenance of the bust. An example is shown in Fig. 4 and Fig. 5, which are a backscattered electron image and corresponding X-ray maps, respectively. The most interesting results from these types of analyses were the preponderance of talc (magnesium silicate) particles found at the surface, indicating that this bust may have been fabricated from or used as a mold for others, as talc is a commonly used release agent. Of even more interest were the numerous particles rich in $\mathrm{Cd}$ and $\mathrm{S}$. Further semiquantitative EDS were performed on these particles and were found to be pure CdS. Others were determined to be CdSeS. These were attributed to pigments used in the surface finish that was applied to the bust - significant in that these pigments were not in use until at least 100 years later, leading to the conclusion that although this bust may still be attributed to Houdon (his signature is on it and dated 1790), he could not have applied the finish. 


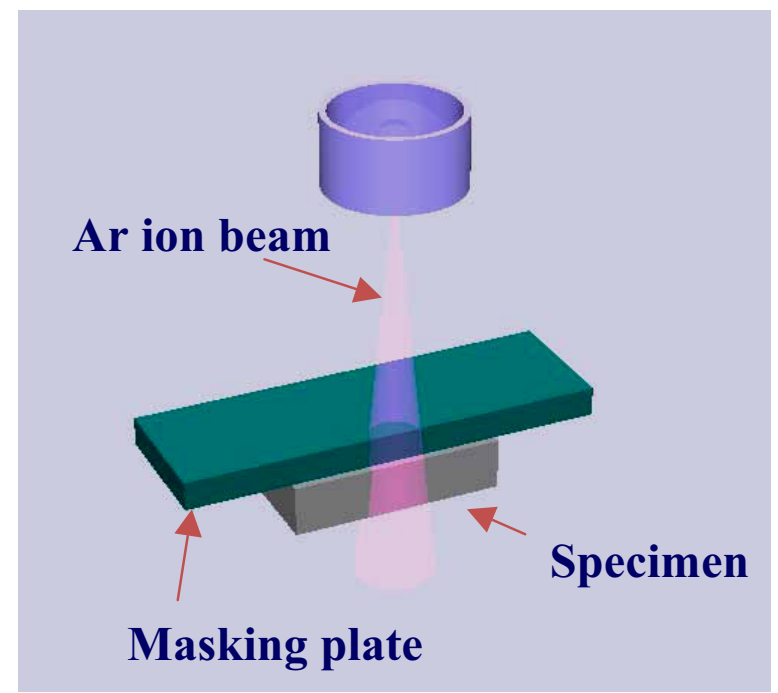

Figure 1: Schematic diagram of ion beam cross section polisher

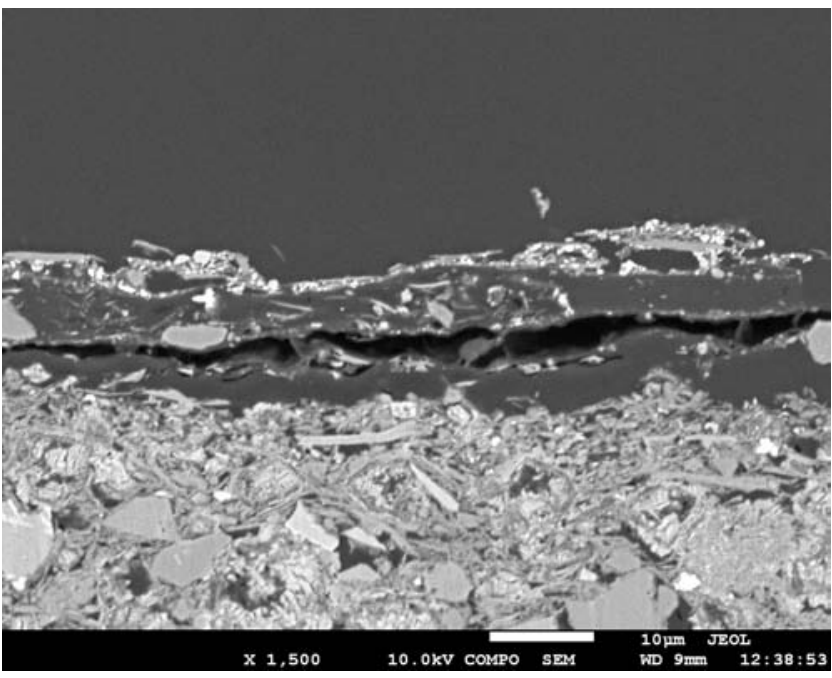

Figure 3: Backscattered electron image of cross section polished surface fragment from Lafayette bust.

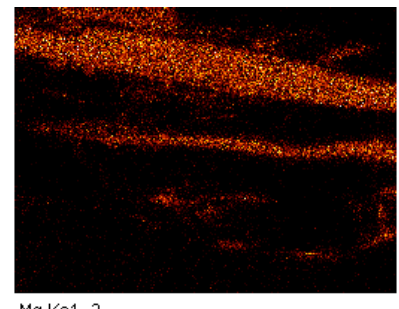

Mg Ka1_2

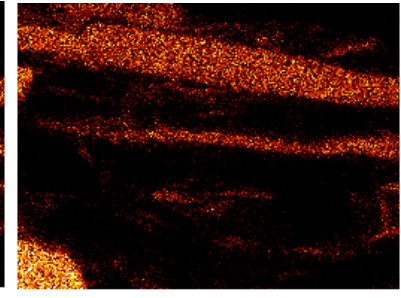
Si Ka1

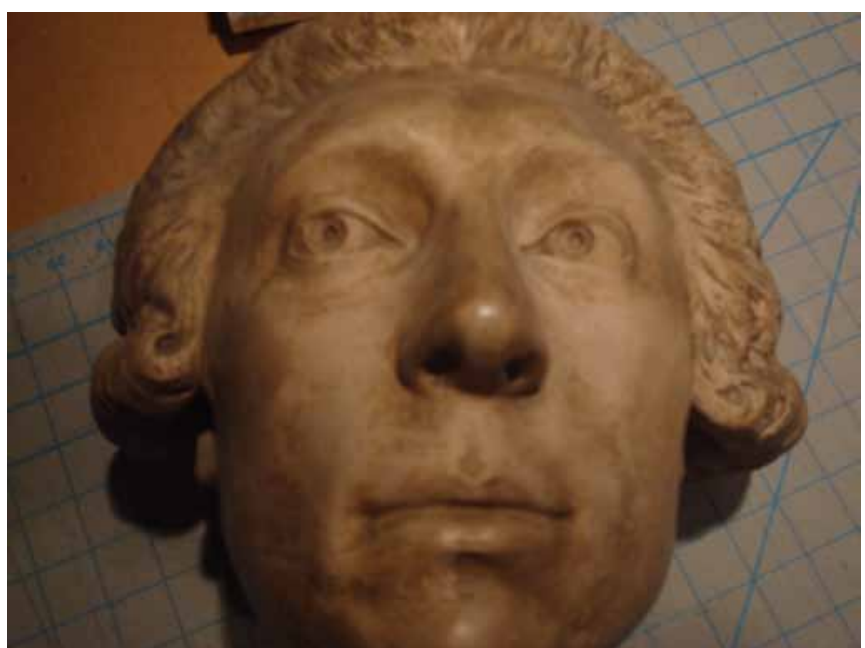

Figure 2: Bust of the Marquis de Lafayette attributed to Jean-Antoine Houdon

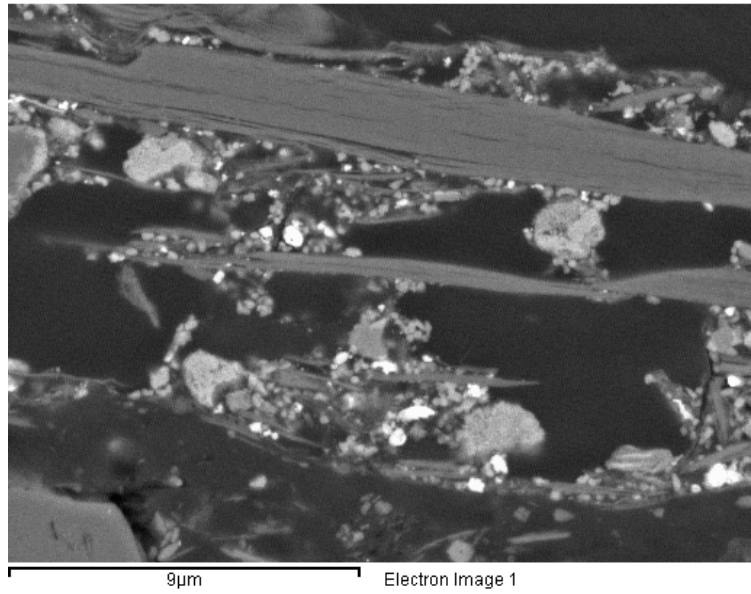

Figure 4: Higher magnification showing detail at surface of fragment. X-ray maps from this region are shown in Fig. 5.
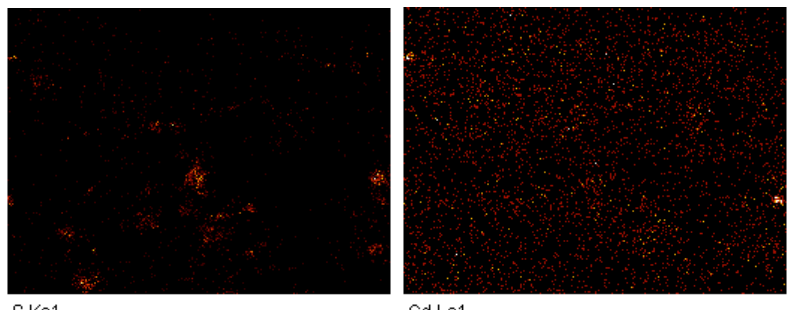

CdLa1

Figure 5: X-ray maps from the area shown in Fig. 4. From left to right are the maps for: $\mathrm{Mg}$, Si, S and Cd. 